\title{
Adrenocortical system activity in alloxan-resistant and alloxan-susceptible Wistar rats
}

\author{
Vera G. Selyatitskaya, Natalya A. Palchikova*, Natalya V. Kuznetsova \\ Siberian Branch of Russian Academy Medical Sciences, Scientific Centre of Clinical and Experimental Medicine, Novosibirsk, Rus- \\ sia; ${ }^{*}$ Corresponding Author: labend@mail.ru, labend@,soramn.ru
}

Received 24 January 2012; revised 29 February 2012; accepted 20 March 2012

\begin{abstract}
In the dynamics of the disease development, diuresis and glycosuria increase in alloxan-susceptible rats, while in alloxan-resistant rats the increase in the values of these indices is expressed to a lesser extent, and they begin to decrease by day 8 of the disease. In alloxansusceptible rats, the mass index of adrenal gland is increased, and that of thymus is decreased and corticosterone concentration in blood, adrenal gland and urine as well as alanine and aspartate aminotransferase activities in liver are increased; in alloxan-resistant rats the values of these indices do not differ from those of rats of the control group.
\end{abstract}

Keywords: Experimental Diabetes;

Alloxan-Resistant and Alloxan-Susceptible Rats;

Adrenocortical System Activity; Corticosteroids

Concentration in Urine

\section{INTRODUCTION}

It is known from literature that at modeling diabetes mellitus type 1 (DM-I) using alloxan selectively affecting $\beta$-cells of the islets of Langerhans, experimental animals from heterogeneous groups $[1,2]$ and linear animals $[3,4]$ display different susceptibilities to the diabetogenic effect of alloxan. The authors attribute this phenomenon to differences in the oxidative status and the activity of antioxidant systems of animals.

Differences in lipid profiles and liver glycogen concentrations, oxidative stress parameters and the activities of antioxidant system enzymes as well as the morphology of beta cells of pancreatic islets have been demonstrated between alloxan-susceptible and alloxan-resistant Wistar rats [1].

Insulin deficiency in the organism is of primary importance in the development of severe metabolic disturbances at DM-I [5]. At the same time, some obtained results show that glucocorticoid hormones can play a negative role in exacerbating the severity of experimental DM-I, their excess secretion by adrenal into the bloodstream resulting in the stimulation of gluconeogenesis processes in the target tissues [6,7].

The aim of this work was to evaluate adrenocortical system (ACS) activity in the dynamics of DM-I development in alloxan-susceptible and alloxan-resistant Wistar rats.

\section{MATERIALS AND METHODS}

\subsection{Experimental Animals and Diabetes Induction}

Adult male Wistar rats obtained from the vivarium of the Institute of Cytology and Genetics SB RAS. The animals were kept in individual cages on the standard diet of the vivarium with access to food and water $a d$ libitum.

The study was approved by the Scientific Centre of Clinical and Experimental Medicine Siberian Branch RAMS (SCCEM SBRAMS) Animal Research Ethics Committee.

Experimental diabetes in rats was simulated by a single intraperitoneal injection of alloxan (Sigma) dissolved in saline solution at a dose of $170 \mathrm{mg} / \mathrm{kg}$ of bodyweight after 18 hours of starvation, whereas the control group received only saline injection [8].

\subsection{Material Collection}

Daily urine samples were collected from rats, and their diurnal diuresis and glycosuria levels were measured during 2 - 10 days after alloxan administration. Urine aliquot was frozen to determine the concentrations of progesterone, DHEA-S and non-metabolized corticosterone.

The rats were withdrawn from the experiment 2 weeks after alloxan administration by decapitation and blood and tissue samples were collected. Bodyweight, the weights of kidneys, thymus, lipid depots (epididymal and 
retroperitoneal fat) and adrenal were measured, and the relative weight of organ/adipose tissue per $100 \mathrm{~g}$ of bodyweight (BW) was calculated (mass index).

The blood was centrifuged and the serum stored at $-20^{\circ} \mathrm{C}$ to determine the concentrations of glucose, immunoreactive insulin (IRI) and corticosterone.

Adrenals were homogenized in phosphate buffer $(0.1$ $\mathrm{M}, \mathrm{pH}$ 7.4). Homogenates were centrifuged and the supernatant stored at $-20^{\circ} \mathrm{C}$ to determine the corticosterone content.

\subsection{Biochemical Measurement}

Glucose concentrations in serum and urine were determined by the enzyme method using GLU kits (BioCon). Alanine aminotransferase (ALT, E.C. 2.6.1.2) and aspartate aminotransferase (AST, E.C. 2.6.1.1) activities and protein content in hepatic tissue were determined using BioCon kits.

\subsection{Measurement of Hormone Concentrations in Urine, Blood and Adrenal}

The concentration of IRI in serum was determined by the radioimmune kit (rio-INS-PG-125I "HOPIBOH NASB", Minsk); progesterone and DHEA-S concentrations in urine were determined by Progesterone-ELISA and DHEA-S-ELISA kits (XEMA, Moscow); corticosterone concentrations in serum, urine and adrenal were measured by the radioimmune method using $[1,2,6$, 7-3H]-Corticosterone (Amersham) and Corticisterone antiserum (Sigma-Aldrich).

From a biological material steroids were extracted with ethyl acetate $(1: 10 \mathrm{vol} / \mathrm{vol})$. The extracts were evaporated to dryness and the residue was dissolved in assay buffer.

\subsection{Statistical Analysis}

Results were expressed as mean \pm SEM. Statistical differences were evaluated using the Kruskal-Wallis ANOVA test followed by the Mann-Whitney test [9]. All analyses were performed using the Statistica 6.0 application package (Statsoft, USA) Differences were considered statistically significant at $P<0.05$.

\section{RESULTS}

Experimental animals were divided into two groups by blood glucose level 2 weeks after alloxan administration: the first one with glucose level 4-fold exceeded this value in rats of the control group, the second one with glucose level exceeding the control level by 1.3 times. As the expression of glycemia reflects the severity of diabetes mellitus, the first group was defined as alloxan-suscentible (ALS), and the second as alloxan-resistant (ALR) rats (Table 1).

The concentration of IRI in serum was 2 times lower in both ALS and ALR rats than in control animals.

Bodyweight and the mass index of lipid depots decreased, and the mass index of kidney increased in ALS rats to a greater extent than in ALR animals.

Table 2 presents the results of determining ACS activity and metabolic effects of corticosterone in ALS and ALR rats.

In ALS rats, the concentration of blood corticosterone, the main glucocorticoid hormone in this animal species, was 1.7 higher than in rats of the control group. The concentration of this hormone in adrenal glands was also 1.5 times higher. In ALR rats, the values of the above indices did not differ from the corresponding values in rats of the control group. The adrenal mass index was also more increased in ALS rats than in ALR rats as compared to this index value in rats of the control group.

AST and ALT activities in the liver of ALS rats were 1.8 - 1.9 times higher as compared to the values of these indices in control rats.

The mass index of thymus was 1.5 times lower in ALS rats than in the control animals.

Table 3 presents the results of determining the values of urine volume anl gycosuria level in the rats' daily urine collected during the first 10 days after alloxan administration.

Expressed glycosuria was observed in experimental

Table 1. Evaluation of diabetes severity after alloxan administration to experimental rats.

\begin{tabular}{|c|c|c|c|}
\hline & Control $(n=40)$ & $\operatorname{ALS}(n=33)$ & $\operatorname{ALR}(n=14)$ \\
\hline Serum glucose, $\mathrm{mmol} / \mathrm{L}$ & $6.36 \pm 0.13$ & $28.85 \pm 2.93^{* *}$ & $8.49 \pm 0.53^{* * \#+\#}$ \\
\hline IRI, pmol/L & $130.2 \pm 8.9$ & $53.3 \pm 7.7^{* *}$ & $60.8 \pm 3.7^{* *}$ \\
\hline Bodyweight, g & $307.1 \pm 11.3$ & $234.7 \pm 7.1^{* *}$ & $288.6 \pm 9.2^{\# \#}$ \\
\hline Mass index of kidney, $g / 100 \mathrm{~g}$ of $\mathrm{BW}$ & $0.30 \pm 0.01$ & $0.56 \pm 0.02^{* *}$ & $0.33 \pm 0.01^{* \#}$ \\
\hline Mass index of lipid depots, $\mathrm{g} / 100 \mathrm{~g}$ of BW & $2.57 \pm 0.11$ & $0.70 \pm 0.08^{* *}$ & $2.11 \pm 0.16^{* \#}$ \\
\hline
\end{tabular}

Significantly different from control $\left({ }^{*} p<0.05,{ }^{* *} p<0.01\right)$; Significantly different from ALS $\left({ }^{\#} p<0.05,{ }^{\# \#} p<0.01\right)$. 
Table 2. Adrenocortical system activity in ALS and ALR rats.

\begin{tabular}{|c|c|c|c|}
\hline & Control $(n=40)$ & $\operatorname{ALS}(n=33)$ & $\operatorname{ALR}(n=14)$ \\
\hline Serum corticosterone, $\mathrm{nmol} / \mathrm{L}$ & $207.9 \pm 32.0$ & $357.7 \pm 27.8^{* *}$ & $214.3 \pm 20.3^{\# \#}$ \\
\hline Adrenal corticosterone, $\mathrm{pmol} / \mathrm{mg}$ of tissue & $46.3 \pm 4.8$ & $70.4 \pm 6.9^{*}$ & $45.3 \pm 6.6$ \\
\hline Mass index of adrenal, $\mathrm{mg} / 100 \mathrm{~g}$ of BW & $7.07 \pm 0.25$ & $11.66 \pm 0.43^{* *}$ & $8.85 \pm 0.31^{* * \# \#}$ \\
\hline AST activity in liver, U/g of protein & $431.5 \pm 30.2$ & $792.8 \pm 64.1^{* *}$ & $468.8 \pm 39.5^{\# \#}$ \\
\hline ALT activity in liver, $\mathrm{U} / \mathrm{g}$ of protein & $206.4 \pm 26.3$ & $402.9 \pm 35.5^{* *}$ & $197.4 \pm 12.3^{\# \#}$ \\
\hline Mass index of thymus, $\mathrm{g} / 100 \mathrm{~g}$ of BW & $0.14 \pm 0.01$ & $0.09 \pm 0.01^{* *}$ & $0.16 \pm 0.01^{\# \#}$ \\
\hline
\end{tabular}

Significantly different from control $\left({ }^{*} p<0.05,{ }^{* *} p<0.01\right)$; Significantly different from ALS $\left({ }^{\#} p<0.05,{ }^{\# \#} p<0.01\right)$.

Table 3. The dynamics of diabetes development in ALS and ALR rats.

\begin{tabular}{|c|c|c|c|c|}
\hline No. & Animals & Days after alloxan administration & Diuresis, $\mathrm{ml}$ & Glycosuria, g/day \\
\hline 1 & Control & & $8.2 \pm 0.6$ & $0.002 \pm 0.001$ \\
\hline 2 & & Days 2 - 4 & $64.6 \pm 5.3$ & $3.8 \pm 1.4$ \\
\hline 3 & ALS & Days $5-7$ & $102.2 \pm 5.0$ & $10.8 \pm 1.5$ \\
\hline 4 & & Days 8 - 10 & $123.7 \pm 4.0$ & $10.3 \pm 0.5$ \\
\hline 5 & & Days 2 - 4 & $13.5 \pm 2.6$ & $2.2 \pm 0.9$ \\
\hline 6 & ALR & Days 5 - 7 & $17.1 \pm 2.3$ & $0.52 \pm 0.25$ \\
\hline \multirow[t]{2}{*}{7} & & Days $8-10$ & $9.6 \pm 2.4$ & $0.02 \pm 0.01$ \\
\hline & & $P$ & $\begin{array}{c}1-2,3,4<0.01 \\
3-2,4<0.01 \\
5-2<0.01\end{array}$ & $\begin{array}{c}1-2,3,4<0.01 \\
3-2<0.01\end{array}$ \\
\hline
\end{tabular}

rats of both groups on days 2 - 4 after alloxan administration. In animals of the control group glucose excretion with daily urine was at the level of thousandth of a gram, whereas in rats that received alloxan injection it increased to a few grams per day.

Table 4 presents the results of determining the concentrations of non-metabolized progesterone, corticosterone and DHEA-S in the rats' daily urine collected during the first 10 days after alloxan administration.

Soon after alloxan administration, progesterone, DHEA-S and corticosterone concentrations in urine of ALS rats were significantly higher than those in control and ALR rats. During the next days, the changes revealed in steroid excretion in ALS rats became more pronounced, and 8 - 10 days after alloxan administration the concentrations of progesterone, DHEA-S and corticosterone were by $2.1,2.7$ and 8.3 times higher than in control animals, respectively. In ALR rats, the values of these indices returned to the control levels within the time mentioned.

\section{DISCUSSION}

The changes in the mass indices of kidney and adipose tissue (Table 1) indicate more expressed metabolic disorders in organs and tissues of ALS rats. However, in spite of a significant difference in glycemia, the serum immunoreactive insulin concentrations were equally decreased in both ALS and ALR rats. It can be supposed that the heterogeneity of experimental animals by the susceptibility to the diabetogenic effect of alloxan is associated not only with the expression of disturbances in the structure and function of the pancreatic islet apparatus of rats caused by it but also with the effects of other hormones enhancing the metabolic effects of insulin deficiency in the organism. These include glucocorticosteroid hormones enhancing glucose synthesis in gluconeogenesis reactions $[10,11]$.

Increased corticosterone concentrations in blood and adrenal glands of ALS rats are indicative of the enhancement of this hormone synthesis (Table 2). The increase in the concentration of the physiologically active form of corticosterone in ALS rats' blood is evidenced by elevated activity of liver enzymes participating in reactions of amino acids involvement in gluconeogenesis (Table 2) [12-14]. As the thymolythic effect is a wellknown consequence of a long-term increase in the concentrations of glucocorticoid hormones in blood $[15,16]$, 
Table 4. Daily excretion of steroids with urine in ALS and ALR rats in the dynamics of development of alloxan-induced diabetes.

\begin{tabular}{|c|c|c|c|c|c|}
\hline No. & Animals & Days after alloxan administration & Progesterone pmol/day & DHEA-S nmol/day & Corticosterone nmol/day \\
\hline 1 & Control & & $120.4 \pm 14.3$ & $0.86 \pm 0.05$ & $0.66 \pm 0.05$ \\
\hline 2 & & Days 2 - 4 & $236.6 \pm 33.6$ & $2.66 \pm 0.26$ & $1.42 \pm 0.32$ \\
\hline 3 & ALS & Days 5 - 7 & $226.6 \pm 59.6$ & $2.95 \pm 0.23$ & $4.48 \pm 0.74$ \\
\hline 4 & & Days 8 - 10 & $250.0 \pm 47.7$ & $2.35 \pm 0.23$ & $5.50 \pm 0.30$ \\
\hline 5 & & Days 2 - 4 & $38.7 \pm 10.8$ & $1.08 \pm 0.15$ & $0.90 \pm 0.19$ \\
\hline 6 & ALR & Days 5 - 7 & $93.2 \pm 33.3$ & $0.93 \pm 0.18$ & $0.91 \pm 0.14$ \\
\hline \multirow[t]{2}{*}{7} & & Days $8-10$ & $114.1 \pm 28.3$ & $0.72 \pm 0.20$ & $0.45 \pm 0.15$ \\
\hline & & $P$ & $\begin{array}{c}2-1<0.01 \\
2-3,4<0.05\end{array}$ & $\begin{array}{c}1-2,3,4<0.01 \\
2,3,4-5,6,7<0.01\end{array}$ & $\begin{array}{c}1-2<0.05 \\
1-3,4<0.01 \\
2-3<0.01 \\
3-4<0.05\end{array}$ \\
\hline
\end{tabular}

the thymus mass index was also measured. Its value was lower in ALS rats as compared to the control level (Table 2). Thus, the obtained results show that the severity of diabetes in rats after alloxan administration is associated with the degree of ACS activation.

There is evidence that the diabetogenic effect of alloxan is associated with the activation of the processes of formation of activated oxygen forms in $\beta$-cells of the pancreatic islets of Langerhans, which results in their death and, therefore, reduces insulin concentration in blood [17]. As the decrease in immunoreactive insulin concentration was equally manifested in the blood of rats with severe and mild diabetes (Table 1), we can speak about equal expression of affection of the pancreatic islet apparatus by alloxan in experimental rats of both groups. Therefore, it can be supposed that ALS rats have initially increased ACS reactivity to the effect of metabolic stressors, which can include metabolism disorders characteristic of diabetes.

The dynamics of diabetes development soon after alloxan administration to rats was studied to test this hypothesis (Tables 3, 4). The results of this study also confirmed the above-mentioned opinion of comparable affection of the pancreatic islet apparatus in experimental rats of both groups after alloxan administration.

In [18] it was shown that rats highly resistant and low resistant to hypoxia have different ACS reactivities (by corticosterone level in blood) in response to acute hypoxia. In [19] it was shown that 5 and more weeks after streptozotocin administration Wistar rats can be divided into two groups: those with stable diabetes or those with the subsequent reversal of hyperglycemia and clinical signs of diabetes. The authors found an association between the presence of two variants of the course of streptozotocin-induced diabetes in rats with different degrees of resistance of pancreatic islet tissue to toxic damage and the subsequent compensatory regeneration. This mechanism of formation of two variants of the course of experimental diabetes and our hypothesis about the role of ACS reactivity in determining diabetes severity in rats do not contradict one another but rather complement each other. Based on the results of morphological examination of spleen, the authors of [19] have shown that the suppression of the functional activity of lymphoid cells and immune dysregulation are observed in rats with the stable course of diabetes. These data agree with our results on an expressed decrease in the mass index of thymus in ALS rats and also indirectly indicate ACS activation as it is glucocorticoid hormones that have a pronounced ability to suppress the immune system activity [20].

\section{CONCLUSION}

The induction of diabetes mellitus type 1 by alloxan reduces immunoreactive insulin level in blood and increases ACS activity in experimental animals. In ALS rats, ACS activation is pronounced and further increases in the disease dynamics, which is manifested not only as increased corticosterone concentration in adrenal glands, blood and urine but also as enhanced effect of glucocortisteroid hormones at the level of target tissues, in particular, in the liver where the activity of gluconeogenesis enzymes increases. In ALR rats, ACS activation is significantly lower and is revealed only during the first days of the disease. Therefore, the severity of alloxan-induced diabetes is associated with the expression of ACS activation, which suggests an important role this hormone system reactivity in the mechanisms of developing decompensation of diabetes mellitus type 1 after initial disturbance of insulin-producing function of the pancreatic 
islets of Langerhans.

\section{REFERENCES}

[1] Behr, G.A., da Silva, E.G., Ferreira, A.R., Cerski, C.T., Dal-Pizzol, F. and Moreira, J.C. (2008) Pancreas betacells morphology, liver antioxidant enzymes and liver oxidative parameters in alloxan-resistant and alloxansusceptible Wistar rats: A viable model system for the study of concepts into reactive oxygen species. Fundamental \& Clinical Pharmacology, 22, 657-666. doi:10.1111/j.1472-8206.2008.00628.x

[2] Jain, D.K. and Arya, R.K. (2011) Anomalies in alloxaninduced diabetic model: It is better to standardize it first. Indian Journal of Pharmacology, 43, 91. doi:104103/0253-7613.75684.

[3] Mathews, C.E. and Leite, E.H. (1999) Constitutive differences in antioxidant defense status distinguish alloxanresistant and alloxan-susceptible mice. Free Radical Biology and Medicine, 27, 449-455. doi:10.1016/s.0891-5849(99)00084-2

[4] Mathews, C.E. and Leiter, E.H. (1999) Resistance of $\mathrm{ALR} / \mathrm{Lt}$ islets to free radical-mediated diabetogenic stress is inherited as a dominant trait. Diabetes, 48, 2189-2196. doi:10.2337/diabetes.48.11.2189

[5] Lowy, C. and Williams, E.D. (1967) The pancreas and diabetes mellitus. Postgraduate Medical Journal, 43, 5160. doi:10.1136/pgmj.43.495.51

[6] Chan, O., Inouye, K. and Riddell, M.C. (2003) Diabetes and hypothalamo-pituitary-adrenal (HPA) axis. Minerva Endocrinology, 28, 87-102.

[7] Selyatitskaya, V.G., Cherkasova, O.P., Pankina, T.V. and Palchikova, N.A. (2009) Functional state of adrenocortical system in rats with manifest alloxan-induced diabetes mellitus. Bulletin of Experimental Biology and Medicine, 146, 708-710. doi:10.1007/s10517-009-0393-6

[8] Palchikova, N.A., Selyatitskaya, V.G. and Shorin, Yu. P. (1987) Quantitative evaluation of the sensitivity of experimental animals to the diabetogenic effect of alloxan. Problemy Endokrinologii, 33, 65-68.

[9] Glantz, S. (1999) Primer of Biostatistics. Practica, Moscow.

[10] Sistare, F.D. and Haynes, R.C. Jr. (1985) Acute stimulation by glucocorticoids of gluconeogenesis from lactate/ pyruvate in isolated hepatocytes from normal and adrenalectomized rats. The Journal of Biological Chemistry, 260,
12754-12760.

[11] Agius, L., Chowdhury, M.H. and Alberti, K.G. (1986) Regulation of ketogenesis, gluconeogenesis and the mitochondrial redox state by dexamethasone in hepatocyte monolayer cultures. The Biochemical Journal, 239, 593601.

[12] Yabaluri, N. and Bashyam, M.D. (2010) Hormonal regulation of gluconeogenic gene transcription in the liver. Journal of Biosciences, 35, 473-484. doi:10.1007/s12038-010-0052-0

[13] Horio, Y., Tanaka, T., Taketoshi, M., Uno, T. and Wada, H.J. (1988) Rat cytosolic aspartate aminotransferase: Regulation of its mRNA and contribution to gluconeogenesis. Journal of Biochemistry, 103, 805-808.

[14] Anemaet, I.G., Meton, I., Salgado, M.C., Fernández, F. and Baanante, I.V. (2008) A novel alternatively spliced transcript of cytosolic alanine aminotransferase gene associated with enhanced gluconeogenesis in liver of Sparus aurata. The International Journal of Biochemistry \& Cell Biology, 40, 2833-2844. doi:10.1016/j.biocel.2008.05.018

[15] Herold, M.J., McPherson, K.G. and Reichardt, H.M. (2006) Glucocorticoids in T cell apoptosis and function. Cellular and Molecular Life Sciences, 63, 60-72. doi:10.1007/s00018-005-5390-y

[16] Kelly, F.J., and Goldspink, D.F. (1984) Age-related growth and protein turnover in the thymus of normal and glucocorticoid-treated rats. European Journal of Biochemistry, 138, 623-627. doi:10.1111/j.1432-1033.1984.tb07960.x

[17] Lenzen, S. (2008) The mechanisms of alloxan- and streptozotocin-induced diabetes. Diabetologia, 51, 216-226. doi:10.1007/s00125-007-0886-7

[18] Grek, O.R., Efremov, A.V. and Grek, O.O. (2002) Hormone level and metabolism of xenobiotics in rats with various phenotype of resistance to hypoxia. Eksperimental'naia I Klinicheskaia Farmakologiia, 65, 53-55.

[19] Zakir'ianov, A.R., Plakhotnii, M.A., Onishchenko, N.A., Volodina, A.V., Klimenko, E.D., Kobozeva, L.P., Michuskaia, A.B. and Pozdniakov, O.M. (2007) Diabetic complications in rats in long-term modeling of type I diabetes mellitus. Patologicheskaia Fiziologiia I Eksperimental'naia Terapiia, 4, 21-25.

[20] Costa-Pinto, F.A. and Palermo-Neto, J. (2010) Neuroimmune interactions in stress. Neuroimmunomodulation, $\mathbf{1 7}$, 196-199. doi:10.1159/000258722 